\title{
MicroGrid Energy Management Optimization - A Common Platform for Research, Development and Design Tools
}

\author{
Patrick Béguery ${ }^{1}$, Peter Pflaum ${ }^{1}$, Carl Mugnier ${ }^{2}$ \\ ${ }^{1}$ Schneider Electric, Grenoble, France \\ ${ }^{2}$ Alten Technologies, Grenoble, France
}

\begin{abstract}
Energy management solutions for microgrids typically rely on advanced control/optimization methods that can efficiently tackle a complex set of goals and constraints. Simulation tools can greatly contribute in the development and deployment of such solutions.

Unfortunately, most of the existing software are lacking one or more of the following required features: capacity to integrate the real energy management algorithm, open environment for research development and continuous integration, and simple to use graphical user interface for the most repetitive cases.

The simulation environment proposed in this contribution is a Matlab/Simulink based framework for the development of district level models and validation of real energy management algorithms (Software-In-the-Loop test bench), that has been completed by post-processing methods and a GUI to produce a design tool.

Initiated in an European project, the framework has been successfully industrialized and is now used at different levels (sales teams, engineering teams, research and development), maximizing synergies and agile transfer between repetitive cases and new business opportunities.
\end{abstract}

\section{Introduction}

Microgrids are typically composed of multiple Distributed Energy Resources (DER) that must be controlled simultaneously to benefit from the most advantageous energy sources and optimize the use of storage. This requires advanced energy management systems (EMS) which consider variable energy rates, load and production forecasts as well as other constraints like maximum power or user comfort. Model Predictive Control (MPC) has been widely used to address this challenge (Zia, 2018). Indeed, MPC provides both a robust control solution and the capacity to be easily extended to new DER architectures and new constraints.

Due to the complexity of the problem and the algorithm used, it is very difficult to guess the optimal design (e.g. battery size, $\mathrm{PV}$ size, ...) and guarantee the performance for a given customer case without using simulation. A significant number of design tools have been developed to provide a first estimate of what can be a good microgrid solution for a specific customer, one of the most successful being HOMER Pro (Lambert, 2006). However, most of these tools are not using advanced energy management methods (such as MPC) that can guarantee good control performances in every microgrid configuration. And when they use some optimization, like EnergyToolBase (ETB, 2018) or Geli Esyst (EMD, 2013), it is to address simple cases (typically photovoltaic and battery). Furthermore, there is no guarantee that the optimization methods used in these tools are representative of the one that will be deployed on the field. For example, to make calculation faster, some of these tools solve the control problem directly on the full year. This is of course not representative for a real EMS which will only have a few hours of forecast information available.

Demand Charge Management, which is based on reducing the monthly peak power, is a good example of a tariff component which is addressed in a very different way by full year optimization and by a real-life MPC controller using a 24-hour prediction horizon. To deal with this issue, in EnergyToolBase, the user can set two factors -Peak Shaving Efficiency and Peak Shaving Utilization Rate- to correct simulated result into more realistic ones. The default values suggested for these factors clearly highlight the gap between full year and real-time optimization. The issue is that defining the corrective factors is not at all easy and should theoretically be done not only for every control solution, but for every customer case.

To obtain more robust results, it is clearly advantageous to consider tools that simulate the microgrid using the real energy management algorithm. Some of the abovementioned software theoretically allow a commercial energy management algorithm to be integrated through predefined API. However, they are not open environments and such an integration would require working closely with the software developer and lack the required agility to address new features.

Indeed, we see that in many cases, a detailed engineering study will have to address some specific customer requests. Some of them can be managed by preprocessing or post-processing, others require to modify the simulation model or the EMS. To tackle this challenge, our approach consists in the development of an open environment based platform that is used both for research and development in one hand (test, validation of energy management evolution), and design on the other hand (selection of the best architecture, sizing and calibration of the energy management algorithm). 


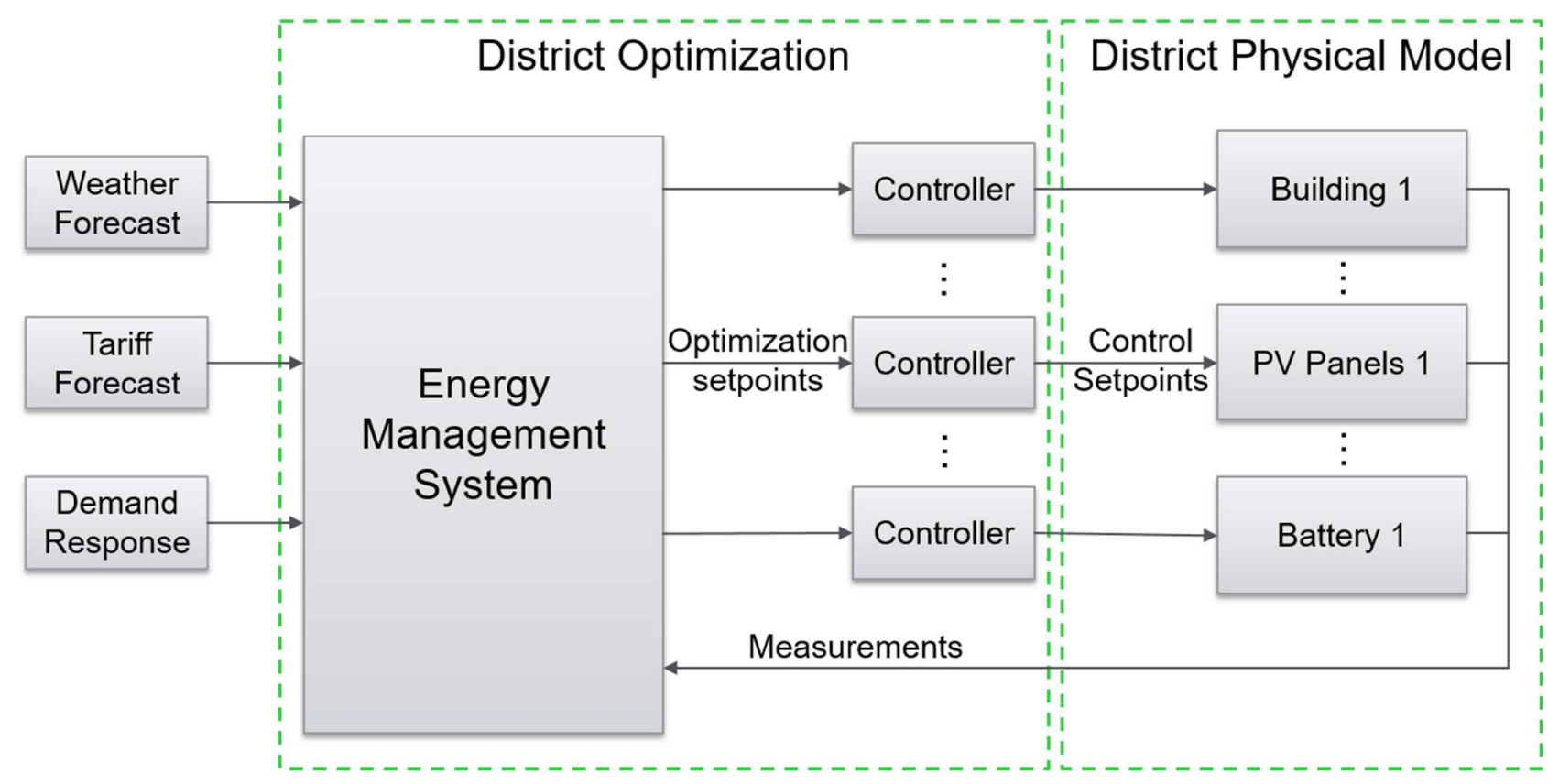

Figure 1: DiSiPl architecture

\section{District Simulation Platform}

Within the scope of the EU AMBASSADOR project, the District Simulation Platform (DiSiPl) has been developed for the validation and performance assessment of multiple cooperative EMS at the district level (Bourry, 2015). The DiSiPl is a research and development platform, but it was built with a few specific features to make it ready to be packaged in full applications.

\section{Specific features}

For each DER considered in the DiSiPl, there is a library of physical models and a corresponding library of typical controllers that can be used in the place of a centralized EMS (to simulate a classical solution without advanced energy management) or as low-level controller to apply EMS strategy (Ex: HVAC emitter control to achieve the target temperature setpoint defined by a building EMS). Each DER model/control library have a template interface with defined named variables to be exchanged with other models.

Instead of the drag-and-drop approach typically used with Simulink, the DiSiPl user writes a data structure in a specific format. This structure will be used by a set of scripts that will automatically build the Simulink model with the architecture shown in Figure 1.

DiSiPl also comes with pre-processors to import weather files (.TMY, .EPW, .PRN format), create calendar and tariff data and build forecasts. After a simulation, results are automatically saved in a standardized format, which allows easy development of post-processing methods.

The current version of the DiSiPl includes libraries for buildings, PV, wind turbine, battery, genset, cogeneration unit, hydrogen fuel cells and electrolysers, thermal distribution network, thermal tank and electrical vehicle charging station. The most commonly used models are:
- Load is very often modeled with predefined profile, either from customer or typical profile like the ones provided by the DOE Commercial Building Models. It is possible to reduced model of building (Beguery, 2015), but the process is complex and not appropriate for commercial use. Integration of whole building models using FMU is under study.

- Photovoltaic production can use import profile from other tools or existing field meter, but it can also be calculated within the tool taking into account global panel irradiation (using irradiation transposition from panel orientation) and cell temperature model. The model delivers AC power (inverter included) and can be curtailed if needed.

- Battery model include SOC dependent maximum charge/discharge powers and losses.

- Genset model is based on linear relation between fuel consumption and electricity power, with minimum power ratio, start cost and minimum running period.

New models for a given DER can easily be added if they follow the template interface defined for their library. The extension of the DER interface or creation of new DER types is a bit more complex as they require modifications of the builder scripts.

Complex DERs including sub-models can also be created. For example, a typical residential model was recently developed. It includes a pre-processor using the CREST generator from Loughborough University (Eoghan, 2016) to create stochastic usage profiles, a domestic hot water tank, a swimming pool pump, an electric vehicle charging station and a fixed battery (Benbrahim, 2018).

\section{Software-In-the-Loop control integration}

One of the DiSiPl's original targets is Software-in-theLoop (SIL) validation of energy management algorithms and evaluation of their global performance. EMS algorithms are wrapped into a Matlab S-function which 
creates the interface between the original code and the DiSiPl. In the scope of the AMBASSADOR project, various EMS from eight partners, using different software technologies including webservices, have been connected to the DiSiPl. More recently, a generic Python-based Energy Flexibility Optimization (EFO) framework has been developed for the commercial offer and integrated into the simulation platform.

\section{Energy Flexibility Optimization Framework}

Model Predictive Control is a popular control technique for microgrid energy management applications (Zia, 2018). This is thanks to its ability to consider forecast information and to deal with multi-variable systems subject to multiple constraints.

The EFO framework has been designed with the objective to easily configure and adapt the MPC controller for a specific microgrid. In fact, while there exist many communalities between different microgrids, each microgrid is unique in the end and needs a customized controller. Being able to configure the MPC controller through a simple configuration file is a crucial step towards a large-scale deployment of microgrid EMS. Furthermore, a unified interface to supply the updated system state and forecast information to the controller at each control instant is of paramount importance for the industrialization of such control solutions.

A typical microgrid control problem generated by the EFO framework is as follows:

$$
\begin{aligned}
\underset{P_{\text {Bat }}}{\operatorname{Minimize}} & \sum_{t=0}^{24} C_{\mathrm{TOU}}(t) \cdot P_{\mathrm{G}}(t) d t+C_{\mathrm{DC}} \cdot \max \left(P_{\mathrm{G}}\right) \\
\text { s.t. } & P_{\mathrm{G}}(t)=P_{\mathrm{L}}^{\mathrm{forec} a s t}(t)+P_{\mathrm{Bat}}(t) \\
& S o C(t+1)=\left\{\begin{array}{lll}
S o C(t)+P_{\mathrm{Bat}}(t) \cdot \Delta t \cdot \eta_{\text {charge }} & \text { if } & P_{\mathrm{Bat}} \geq 0 \\
S o C(t)+P_{\mathrm{Bat}}(t) \cdot \Delta t \cdot \eta_{\text {discharge }} & \text { if } & P_{\mathrm{Bat}}<0
\end{array}\right. \\
& 0 \leq S o C(t) \leq S o C_{\mathrm{max}} \\
& -P_{\mathrm{Bat}, \text { max }} \leq P_{\mathrm{Bat}}(t) \leq P_{\mathrm{Bat}, \max }
\end{aligned}
$$

Note that the underlying microgrid is very simple and consists of a building load and a battery storage system. $P_{G}$ is the grid power, $P_{B a t}$ is the battery power and $P_{L}^{\text {forecast }}$ is the forecasted load power profile. The energy contract of the microgrid consists of variable time-of-use price $C_{T O U}$ and demand charge cost $C_{D C}$ which applies to the maximal power peak. The control variables are the battery power for each time-step in the prediction horizon and the constraints represent the power balance equation in the microgrid and the battery model. Typically, the sampling period in the control problem is 15 minutes and the prediction horizon is 24 hours, which is a reasonable choice, since it allows to anticipate the system behavior over a full day while maintaining a sufficiently fine timestep granularity. Figure 2 shows a typical day-ahead plan obtained from solving the above optimization problem.

As a concluding remark on the MPC controller, it is important to have in mind that its performance strongly depends on the precision of the available forecasts. Although, by default, the DiSiPl uses perfect forecast like other microgrid simulation tool, more realistic simulations are feasible using the right pre-processor or even integrating real forecast algorithms if available.

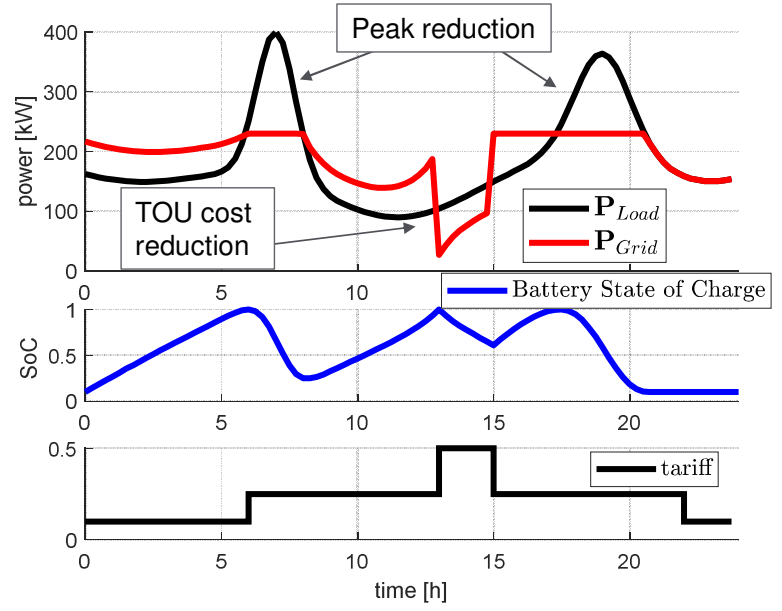

Figure 2: Optimal battery control plan obtained from the MPC control problem at a given time step.

\section{Past use cases}

Thanks to the genericity of the EFO framework, very diverse microgrid configurations have been addressed. To illustrate this genericity, three past use cases which were realized by this framework are described in the following: 1) Microgrids composed of a building load, a PV system, a battery storage system and a cogeneration system. This use case is typical in the United States and the tariff context typically comprises variable time-of-use tariffs and demand charge management.

2) Critical loads supported by Uninterruptable Power Sources (UPS). Each UPS is equipped with a battery storage system and a recent trend is to oversize the battery to use it for peak-shaving or energy cost minimization, additionally to its classic backup function.

3) Multi-energy microgrids such as the Learning Grid by Grenoble which are not only connected to the electricity grid, but also to a district heating network. The coupling between the two energy vectors through a cogeneration system and the additional flexibility thanks to thermal energy storage systems were integrated and managed by the EFO framework, and validated with the DiSiPl.

\section{Integration and validation}

\section{Algorithm integration}

EFO framework flexibility allows fast and robust adaptation of the optimization algorithm to various customers' requirements. However, before deploying the new control system on the real site, it is critical to validate and evaluate its performances. This is done by SoftwareIn-the-Loop validation of EFO within the DiSiPl.

The first step is to create a connector between the simulation platform and the control. A Matlab class is defined with several methods for the optimization execution which include two main functionalities: getting measurements and forecasts to update the optimization problem and solving it to get the optimal control setpoints. In a second step the optimization class methods are integrated into an S-function which provides the interface 
with the Simulink model. The data structure containing the description of controllers and physical models is used to generate the required forecasts and input to the control, and distribute the control output to the relevant DERs.

\section{Validation}

The validation process consists in checking the correct behavior of the algorithm, comparing the performances against other solutions and evaluating the robustness to parameters or input modifications.

During the creation of the algorithm, the scope of its utilization and the associated limitations or constraints are precisely defined. A physical model of a use case that matches the algorithm's target is then created in the DiSiPl. The behavior of the algorithm is assessed through a set of predefined simulations: Summer and Winter typical weeks and full year analysis. The seasonal studies highlight the differences in PV production, load consumption and tariff structure that can disturb the algorithm's behavior. The yearly analysis is used to detect infrequent suboptimal control setpoints and to find their origin. One of the potential causes is the difference between the simple model embedded inside the optimization and the more complex physical model in the DiSiPl. The validation process also supports the verification of compliance with the constraints.

The global performance is then compared to other solutions (different optimization algorithms or simple expert rules). Main metrics are the final energy price and total energy imported from the grid but the computation time or potential issues with the optimization solver can also be studied. A representative full year simulation with a problem resolution at each step will induce several thousands of calls to the optimization solver with various scenarios. This diversity greatly reduces the risk to meet infeasible control problem instances, impossible control setpoints or unexpectedly high solver times when deploying the controller in real life.

The last part of the validation consists in further analysis such as the robustness of the results previously obtained. The major drivers of the algorithm's behavior will be modified: amount of local production, schedule and prices of the energy tariffs, DER sizes, precision of the forecasts. The impact of some specific parameters of the optimization models can also be studied. The simulation can even be used to help in the calibration of some weighting parameters of the optimization problem that are otherwise manually set by the expert.

As an example, a study was run on the addition of genset to the simple Load+PV+Battery case. The genset model requires to introduce binary variables in the control problem which may result in excessive computation times which furthermore are highly volatile from one problem instance to another. One possible solution is to set the "max solver time" variable when calling the solver. The solution seems to work well on a few individual tests of the EFO algorithm. However, full year simulation demonstrate that the global behavior of the control will be greatly impacted by this choice (see Figure 2) and that another solution should be considered.

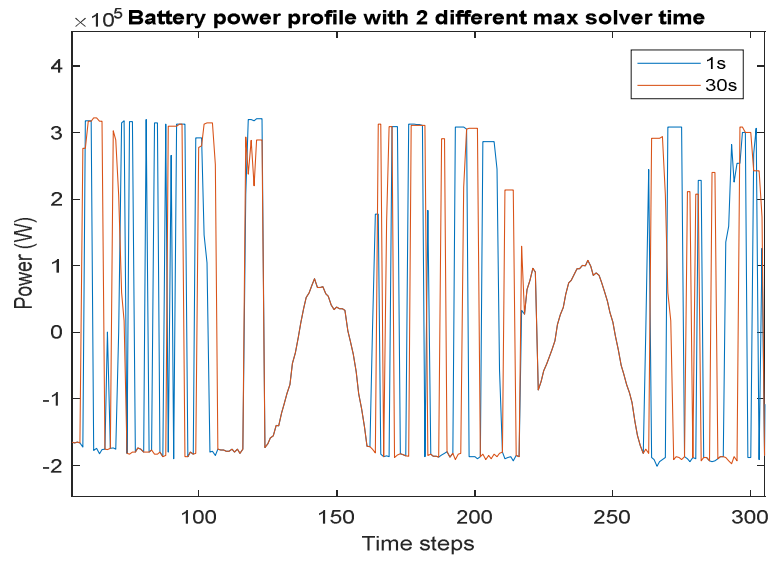

Figure 3: Max solver time impact on optimal strategy.

\section{Design tools}

The DiSiPl is continuously used to validate energy management solutions and research studies on new business cases. It is a very flexible tool but it requires some engineering skills. To allow non-expert Matlab/Simulink users to easily address simple repetitive cases and reduce the time needed to run a design study, it was decided to build a full application around the simulation platform. This software package is named the MicroGrid Design Tool (MGDT).

\section{Project data and functions}

For EMS research and development, it is often enough to compare yearly energy flows and operational expenditure (OPEX) to validate a solution or establish a benchmark. When simulation is to be used for design and sizing purposes, the yearly simulation results must be completed by capital expenditure (CAPEX), operational and maintenance cost, incentives, inflation rates... These data will be used, together with the simulation results, to produce the typical key performance indicators (KPIs) that the customer wants to optimize: Net Present Cost / Value (NPC / NPV), Levelized Cost of Energy (LCOE), Return of Investment (ROI), Payback time... Other indicators will be computed to assess the environmental performance of the grid like the self-consumption, selfproduction, $\mathrm{CO} 2$ emissions, renewable penetration... Furthermore, in a design project, multiple simulations will be run to get reference values and search the optimal size.

To support the development of design tools, a new data structure was proposed. It includes the parameters required to compute KPIs, a baseline data model (the one that is used to build the baseline model in the DiSiPl), customer data and information about the various simulation variants to be run.

Based on this data, a set of functions were developed to launch simulations batch, manage result files, calculate all the KPIs and export results in different formats (including a word report generator). Some interesting challenges with the development of these project functions are:

- Make them generics so that the extension of the tool to a new DER requires little modification of the code.

- Allow user to easily add new incentive formula, new KPIs, new references... 
Table 1: Requirements of a simulation-based application depending on its usage.

\begin{tabular}{|l|c|c|c|c|c|}
\cline { 2 - 6 } \multicolumn{1}{c|}{} & Conviction tool & Sales contact & Early design & Adv. design & R\&D \\
\hline User & Customer & Sales & Sales & Engineer & Researcher \\
\hline Study time & Minutes & Minutes & Hours & Days & Weeks/Months \\
\hline Access & Web & Any, with full GUI & \multicolumn{2}{c|}{ Any, with partial GUI } \\
\hline Real EMS & No & $?$ & Repetitive & Close to repetit. & Any \\
\hline Use case & \multicolumn{2}{|c|}{ Repetitive, simple } & \multicolumn{2}{c|}{ Nice to have } & Not required \\
\hline Use of DB & \multicolumn{2}{c|}{ Mandatory } & \multicolumn{2}{c|}{ Partial } & Yes \\
\hline User extendable & \multicolumn{2}{|c|}{ No }
\end{tabular}

\section{MicroGrid Design Tool}

While developers are typically at ease with a flexible environment like Matlab or Python, a design will most often be performed by people with much less computer skills and much less time to spent on each case. Thus, the development of Graphical User Interface is mandatory. Specifications of such a GUI will depend on the final user targetted, as illustrated in Table 1.

The MicroGrid Design Tool (MGDT) is a Matlab GUI developed upon the DiSiPl for early/advanced design addressing repetitive cases using the real EMS algorithm. Such a tool will be equivalent to HOMER Pro but including advanced optimization methods like EFO. It allows the user to:

- Select a microgrid architecture and control.

- Import photovoltaic and load profiles or create typical ones from weather and building profile databases.

- Create an energy rate (editor).

- Select physical component in database and edit them.
- Create and store incentive functions.

- Run multiple simulations (yearly, 15-minutes timesteps) by changing any model parameter.

- Analyze results through various KPIs and visual analytics.

- Export results in table, power profile or Word report.

MGDT allows the quick and realistic evaluation of a microgrid design for a given customer. It was developed to address typical simple customer cases: load, photovoltaic, battery and genset/cogeneration, connected to a grid with complex tariff including time-of-use (TOU) and demand charge management (DCM). For the typical cases it addresses, the tool was successfully used in several countries.

On the other hand, we see that, in a significant number of projects, there are some requirements that are not covered by the MGDT. Such specific customer request can include complex, condition-based incentives, constraints on the usage of DERs, new revenue stream to be considered in

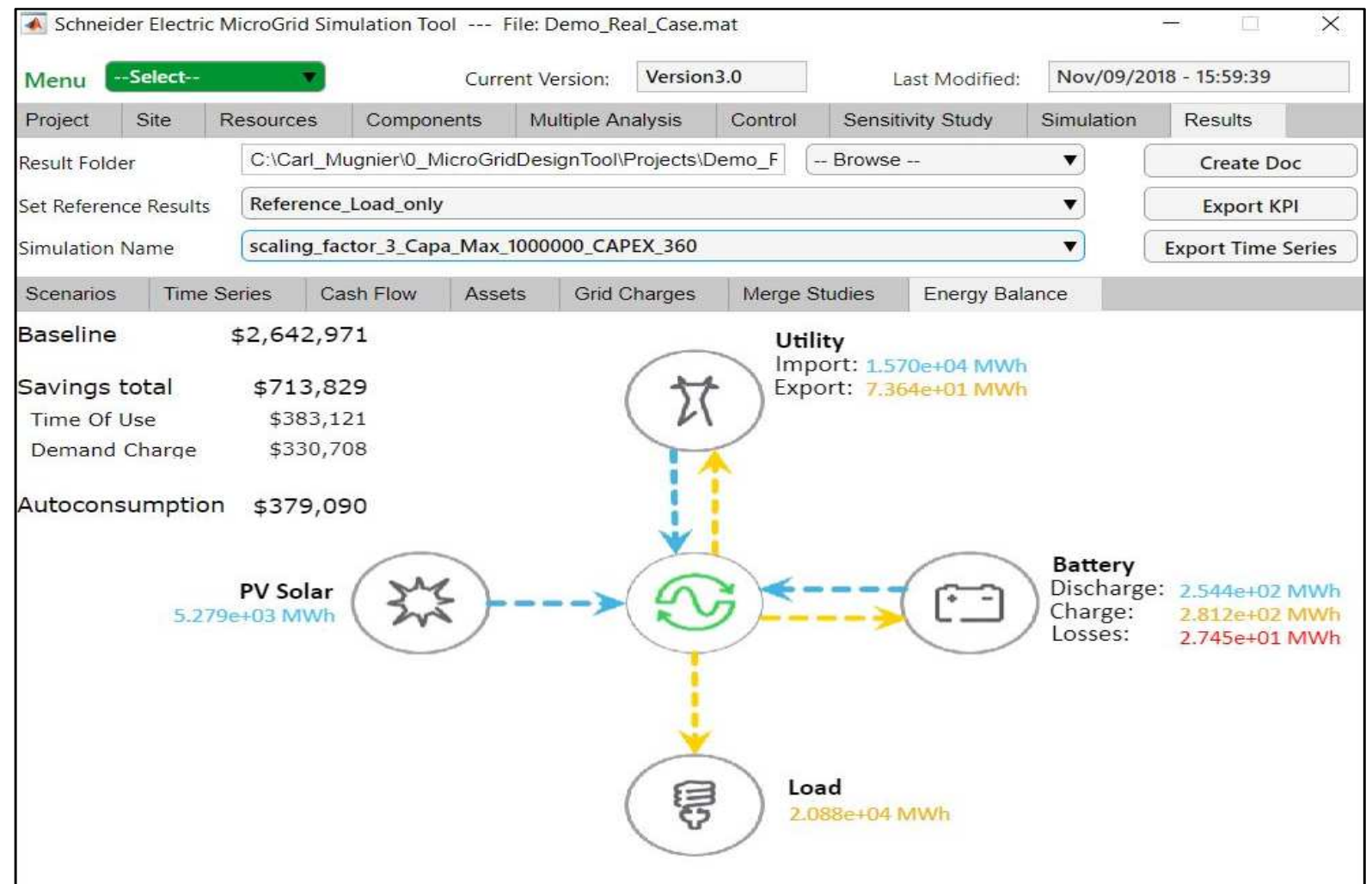

Figure 4: Sample of the MicroGrid Design Tool interface 
the on-line optimization... When using commercial tools, these specific requirements are traditionally done as pretreatment or post-treatment using excel or similar tools. Using multiple tools is not efficient, especially if these calculations have to be run separately for each simulation of the design optimization process. One advantage of MGDT is the fact that it is built on the open Matlab environment. User can directly integrate the pre- or posttreatments in Matlab, or even play with the model and optimization algorithm, within the tool. This allows the study to use the full potential of the MGDT, but also facilitates its potential integration for future re-use.

Of course, proper development of additional code requires a good understanding of the software and should not be done by any sales team. In fact, we have identified three levels of users for our tool:

- Standard users play with the GUI. This requires little software competency but only allows to address the exact repetitive case for which the tool was developed.

- Power users will use typical models and optimization algorithms but will implement some pre- or postprocessing to extend the tool coverage. Pre-formatted templates of some of the most interesting parts are available and new functions can easily be added to the GUI. This includes new KPI calculations, complex incentives development, customization of report...

- Advanced users can modify anything, including models and optimization algorithms, which requires a deep understanding of the tool. It is not guaranteed that the GUI will continue to work and some of the analysis will have to be done manually in Matlab.

\section{Illustrating the framework flexibility}

As already mentioned, a valuable strength of the tool is that the GUI-packaged version of the tool is an upper layer of the platform used for research and development. This enables the expert users to play with the tools at different levels. A project may start with the simple-to-use MGDT, but then can be extended by customer specific features programmed at the DiSiPl level. On the other hand, any new version of the EFO validated on the DiSiPl can be readily integrated in future versions of the MGDT if it is agreed to have the required GUI adaptation performed.

Here are some examples of advanced cases and how they were successully addressed in the last year:

- A US campus with 4 buildings separately connected to the grid, to be equipped with photovoltaic and battery. In islanded mode, all buildings are connected and a backup genset is available. Five models were developed, and an additional calculation was added to assess the statistical autonomy. (Anwar, 2018) describes in details this customer case and how the MGDT was used to provide a specific proposal.

- In the US, some storage incentives have values that are based on the effective percentage of renewable used to charge the battery. This case requires not only a new incentive function to be developed, but also a new constraint to be added to the control problem.
- Another case is a renewable power plant that consider using energy storage to optimize its participation to different utility market. From an optimization point of view, it was just a change of parameters. On the design tool level, there was a few calculations and displays that needed to be corrected and a specific preprocessing to be done for the electricity sale profile.

Each of the above cases have been addressed in a few hours or days, with the final results being shared with the customer using the MGDT GUI, instead of the typical excel sheet used to achieve external post-treatment of classical commercial tools' results. Moreover, most of the ad-hoc developments made for these specific cases were definitively added to the next version of the MGDT.

In parallel with this continuous extension of the typical cases, the platform is also used for more complex, research-oriented investigation, including:

- Learning Grid by Grenoble is a French campus with several buildings, some of them equipped with DER and their own energy management, and all of them connected to a district level which controls an additional battery and a co-generation unit.

- A residential model to study optimization of selfconsumption through control of the hot water tank and kitchen appliance (Benbrahim, 2018) that was later extended to control of swimming pool pump, electrical vehicle charging station and fixed battery.

\section{Simpler tool for pre-sales}

Even though the MGDT was successfully deployed, it proved to be too complex to be used in the early contact, when the target is not to perform a robust proposal but to convince the customer of the proposed solution potential. At this stage, only a few parameters should be easily selected to run a demonstrative study in no more than a few minutes. Furthermore, there is a great interest at this stage, for web based tools which do not require installation and/or licenses.

Achieving the above requirements is not possible with the MGDT/DiSiPl, especially because of Simulink use. On the other hand, running a simplified MPC simulation (one update per day, 365 call to the solver for one year) is already taking too much time. For this reason, a new computation engine was developed based on a set of expert rules that tries to mimic the MPC behavior.

This simple computation engine was based on the same data structure as the MGDT, so that most of the code was reusable. It was packaged with a GUI to address cases like the ones managed by the MGDT, but with simplified parameter settings. The resulting MGDT Lite application targets pre-sale use cases and is deployed either as a standalone application or a web application (the user no more requires a Matlab/Simulink license).

In simple cases, expert rules may achieve a performance close to MPC. However, on more complex cases, the gap might be quite significant. Indeed, as we are no more using the exact EFO algorithm, we meet the same problem as with a commercial tool like ETB or HOMER. 
One advantage however is that our sales tool and engineering tool share the same platform, same data structure and most of analysis functions. This makes comparison very easy and will, in a future version, allow to move from one tool to another with the same model.

To assess the statistical gap between expert rules approach and simplified MPC, a 680 cases study was performed. The customer profiles come from eight of the typical buildings of the DOE Commercial Buildings Database, in 17 typical climates and with the ASHRAE 2013 construction standard (Deru, 2011; Goel, 2014). The study target is to find the optimal photovoltaic production and battery storage that minimizes the Net Present Cost (NPC). Figure 5 shows the distribution between NPC gain calculated with MPC and expert rules. Obviously, MPC is nearly always better, which means that we will not overestimate the microgrid benefit when using expert rules. The mean error on the estimated savings is about $13 \%$, which is acceptable in early stage. However, the error can be much higher in specific conditions: dryer climate have a mean error of about $18 \%$, while it is $23 \%$ on the most complex rate considered and close to $50 \%$ in the worst case.

It is also interesting to note that, as MPC is more efficient in optimizing the OPEX cost, it will find optimal solutions with higher CAPEX. Here also, the gap might be very different depending on the case. On the same study, the mean increase of PV optimal peak power is $17 \%$ (37\% for the battery capacity) when sized with MPC compare to expert rules.

This study confirms that, if a simple approximate tool can be useful in early customer contact, a more realistic one is needed for robust design and performance evaluation of a specific customer microgrid.

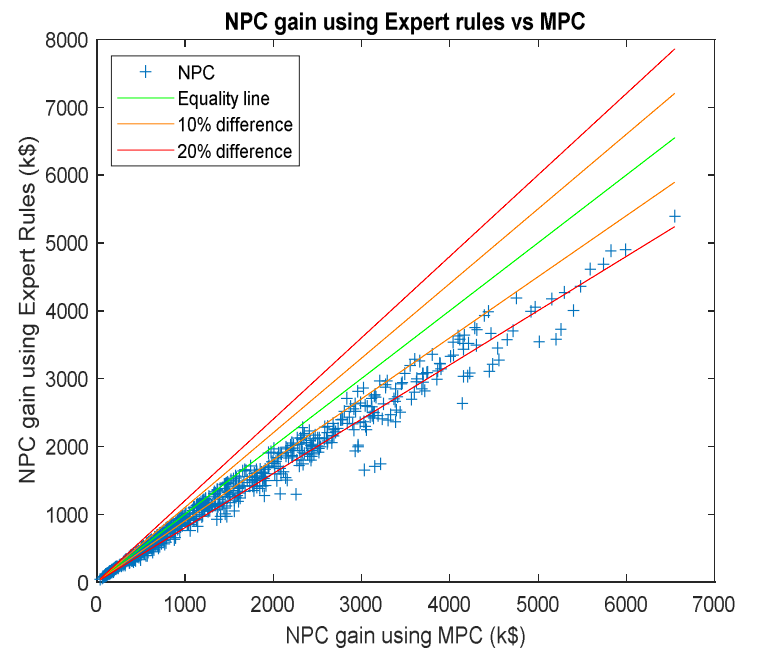

Figure 5 - Impact of using expert rules instead of simplified MPC optimization.

\section{Conclusion and next steps}

Development and maintenance of research and design tools are costly, especially in an unmatured market like microgrids, where there is a clear need to frequently address specific customer requirements. This implies that the simulation tool must be sufficiently open so that an advanced user is able to program additional features (KPIs, visual analytics, create reports automatically using the tool, and even test new algorithm methods), while less advanced users will be able to use the same tool to address more typical cases.

One answer to these challenges was to use the open Matlab and Python environments to develop an optimization code framework, a simulation platform and economic design tools. The DiSiPl is used for SoftwareIn-the-Loop validation of new optimization algorithms developed in the EFO framework, while its GUI packaged version, the MGDT, is used by engineering teams to build commercial propositions. A lite version is also available to run simple sizing studies through a web interface. However, the simplification needed in this tool makes the results less accurate and it is strongly suggested to run a full simulation for any commercial offer.

The current suite of tools offers a very high flexibility between repetitive cases and specific customer cases, which is a key differentiator compared to other commercially available software in the market.

Among the potential extension of the tool is the reusability of the microgrid model during the operational phase. In that case, the model developed during the design phase can become the so-called digital twin of the real microgrid, providing several interesting opportunities for additional digital services:

- In case some parameters of the optimization requires tuning, the simulation can be used to pre-calibrate these parameters.

- Manual comparison between real and simulated results as part of the commissioning phase.

- Automatic comparison can also be considered for performance follow-up (continuous commissioning).

- In case of performance contracting, simulation can be used to remove the bias due to change in weather, usage, energy price hypothesis. This is the suggested approach in Option D of the International Performance Measurement and Verification Protocol (EVO, 2016).

- If case of important context changed, a new optimal solutions can be proposed. This is also possible when new version of the optimization control is released. In both cases, the simulation is used to forecast what will be the benefit for the customer of such a retrofit.

- Finally, the simulation can also provide insights of potential site evolutions by running "what if..." studies similar to the ones run at the design step.

Investigating the potential usage of a digital twin raises a number of new challenges. Real data (customer historical parameters and sensors timeseries) are typically stored in in the cloud. Interfaces will be needed between this data storage and the simulation, both to feed the last one with real data and to store simulation results.

Extending the usage of DiSiPl and MGDT to this new usage is under consideration, thus leveraging the design model development cost and creating new value stream for simulation tools. 


\section{References}

Anwar, A., et al. (2018). Optimal DER Sizing Using Microgrid Design Tool Integrating Model Predictive Control Based Energy Management - A Case Study, Proceeding from IEEE Energy Conservation Congress \& Expo, Portland (USA), 2018.

Benbrahim, Y., et al. (2018). Impact of load management on PV self-consumption in residential sector, Schneider Electric White Paper, 2018.

Beguery, P., et al. (2015). Multi-Zone Reduced Building Models Automated Generation for District Simulation, Proceedings from Building Simulation, Hyderabad (India), 2015.

Bourry, F., et al. (2015). Simulation for the evaluation of energy management algorithms at the district level Example of use case from the AMBASSADOR project, Proceedings from PowerTech, Eindhoven (The Netherlands), 2015.

Deru, M. et al. (2011). U.S. Department of Energy Commercial Reference Building Models of the National Building Stock, NREL Technical Report TP5500-46861, 2011.

EMD (2013). Battery and E-cars as a storage opportunity in energyPRO, EMD / EnergyPRO "How to guide", 2013.

Eoghan McKenna and Murray Thomson (2016). Highresolution stochastic integrated thermal-electrical domestic demand model. Applied Energy, 165-445.

ETB (2018). Energy Storage - Resource guide \& User instructions, ETB Ressource Guide, 2018.

EVO (2016). Core Concepts - International Performance Measurement and Verification Protocol, EVO 100001:2016.

Goel, S., et al. (2014). Enhancement to ASHRAE Standard 90.1 Prototype Building Models, PNNL Report 23269, 2014.

Lambert, T., et al. (2006). Micropower System Modeling with Homer. In Farret, F., Godoy Simoes, M. Integration of Alternative Sources of Energy, John Wiley \& Sons, New-York (USA).

Olivares, D.E., et al. (2014). Trends in Microgrid Control, IEEE Transactions on Smart Grid (vol. 5, no. 4), 1905-1919.

Pflaum, P., et al. (2014). Comparison of a primal and a dual decomposition for distributed MPC in smart districts, Proceeding from IEEE International Conference on Smart Grid Communications, Venice (Italy), 2014.

Raimondi Cominesi, S., et al. (2015). Two-layer predictive control of a micro-grid including stochastic energy sources, Proceedings from 2015 American Control Conference, Chicago (USA), 2015.
Zia, M. F., et al. (2018). Microgrids energy management systems: A critical review on methods, solutions, and prospects. Applied energy, 2018. 